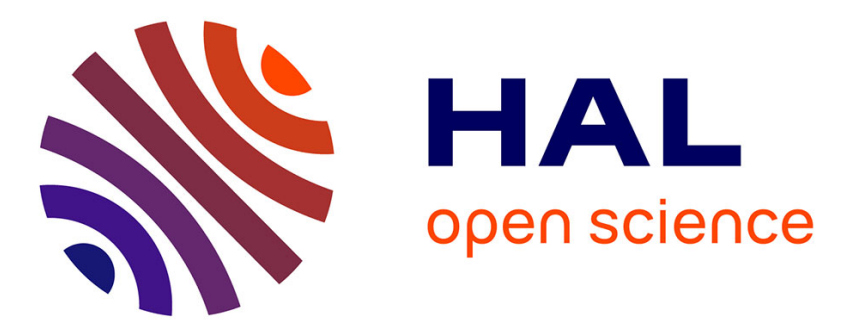

\title{
Bifurcations of emerging patterns in the presence of additive noise
}

Gonzague Agez, Marcel G Clerc, Eric Louvergneaux, René G Rojas

\section{To cite this version:}

Gonzague Agez, Marcel G Clerc, Eric Louvergneaux, René G Rojas. Bifurcations of emerging patterns in the presence of additive noise. Physical Review E , 2013, 87 (4), pp.042919. 10.1103/PhysRevE.87.042919 . hal-01730533

\section{HAL Id: hal-01730533 https://hal.science/hal-01730533}

Submitted on 14 Mar 2018

HAL is a multi-disciplinary open access archive for the deposit and dissemination of scientific research documents, whether they are published or not. The documents may come from teaching and research institutions in France or abroad, or from public or private research centers.
L'archive ouverte pluridisciplinaire HAL, est destinée au dépôt et à la diffusion de documents scientifiques de niveau recherche, publiés ou non, émanant des établissements d'enseignement et de recherche français ou étrangers, des laboratoires publics ou privés. 


\title{
Bifurcations of emerging patterns in the presence of additive noise
}

\author{
Gonzague Agez, ${ }^{1}$ Marcel G. Clerc, ${ }^{2, *}$ Eric Louvergneaux, ${ }^{3, \dagger}$ and René G. Rojas ${ }^{4, \ddagger}$ \\ ${ }^{1}$ Centre d'Elaboration de Matériaux et d'Etudes Structurales, Université Paul Sabatier, 29, rue Jeanne Marvig, \\ BP 94347, 31055 Toulouse Cedex 4, France \\ ${ }^{2}$ Departamento de Física, Facultad de Ciencias Físicas y Matemáticas, Universidad de Chile, Casilla 487-3, Santiago, Chile \\ ${ }^{3}$ Laboratoire de Physique des Lasers, Atomes et Molécules, UMR CNRS 8523, Centre d'Études et de Recherches Lasers et Applications, \\ Université de Lille 1, 59655 Villeneuve d'Ascq Cedex, France \\ ${ }^{4}$ Instituto de Física, Pontificia Universidad Católica de Valparaiso, Casilla 4059,Valparaiso, Chile \\ (Received 26 April 2012; revised manuscript received 7 February 2013; published 23 April 2013)
}

\begin{abstract}
A universal description of the effects of additive noise on super- and subcritical spatial bifurcations in onedimensional systems is theoretically, numerically, and experimentally studied. The probability density of the critical spatial mode amplitude is derived. From this generalized Rayleigh distribution we predict the shape of noisy bifurcations by means of the most probable value of the critical mode amplitude. Comparisons with numerical simulations are in quite good agreement for cubic or quintic amplitude equations accounting for stochastic supercritical bifurcation and for cubic-quintic amplitude equation accounting for stochastic subcritical bifurcation. Experimental results obtained in a one-dimensional Kerr-like slice subjected to optical feedback confirm the analytical expression prediction for the supercritical bifurcation shape.
\end{abstract}

DOI: 10.1103/PhysRevE.87.042919

PACS number(s): 05.45.-a, 42.65.Sf, 05.40.Ca, 45.70.Qj

\section{INTRODUCTION}

Most of the common transitions observed in nature correspond to a breaking of symmetries. In spatially extended systems, when an ordered pattern emerges from an initially homogeneous state [1], they are called morphogenesis. They occur when a control parameter, such as an external condition or a manageable input, is modified. Such transitions between two macroscopic states phenomenologically correspond to phase transitions in the sense of Landau [2]. On the basis of phase diagrams, bifurcation representations have been used as a conceptual tool to characterize the change of behavior of systems as a function of a control parameter [1]. This universal tool comes from order-parameter equations that describe the dynamics of the critical mode amplitude in the vicinity of the bifurcation [3]. It has been largely used to study pattern formation arising in deterministic systems, such as chemical [4] or catalytic reaction systems [5], gas discharge systems [6], $\mathrm{CO}_{2}$ lasers [7], and liquid crystals [8,9], or emerging from hydrodynamic [10] or electroconvective instabilities (see Refs. [11-15] and references therein), to mention a few. The bifurcation diagrams are extremely relevant for characterizing the instabilities in a simple manner. In particular, bifurcation points are significant until no noise is present in the system under study. On the other hand, in the presence of noise, the bifurcation shapes are modified. Bifurcation points become meaningless and no analytical expression of the mean value of the amplitude as function of noise intensity level of the stochastic equation is obtained directly so the shape of the bifurcation and its characteristics cannot be predicted. Hence, below and above bifurcation point regimes can no longer be treated separately. The bifurcation diagrams then no longer can be used to classify and identify the classes of

\footnotetext{
*marcel@galileo.dfi.uchile.cl

†eric.louvergneaux@univ-lille1.fr

${ }^{\ddagger}$ rene.rojas@ucv.cl
}

normal forms of stochastic systems. In the case of nonextended systems, the influence of noise on bifurcations has been studied using both Fokker-Planck and functional integral methods (see Refs. [16-18] and references therein). More precisely, it has been shown that the stationary probability, Lyapunov exponents, correlation functions, and exit times close to the deterministic bifurcation point vary according to the control parameter and noise intensity level. In the past few decades much effort has focused on understanding the influence of noise on extended systems (see the review in Ref. [19] and references within).

In this article, we give analytical expressions of stochastic super- and subcritical bifurcations obtained when additive noise is present in one-dimensional spatial systems. From these universal expressions, the shapes of the bifurcations, their locations, and their evolutions with the noise intensity level are completely defined. Thus, these expressions still allow the use of bifurcation diagrams to study and characterize classes of stochastic amplitude equations. Namely a cubic and a quintic amplitude equations accounting for stochastic supercritical bifurcation and a cubic-quintic amplitude equation accounting for stochastic subcritical bifurcation are studied. Our predictions are compared with experimental works when they exist, i.e., for the supercritical cases. In these cases, the agreement is excellent.

Our approach follows one of our previous works on stochastic supercritical bifurcation corresponding to a cubic amplitude equation [20]. We extend this first study to subcritical ones and to quintic supercritical ones. More precisely, we provide evidence regarding how the presence of noise drastically changes the shapes of both super- and subcritical deterministic bifurcation diagrams. In each case, the stationary probability distribution for the spatial critical mode is obtained. Then, we deduce, from these distributions, the evolutions of the most probable value of the pattern amplitude from below to above the threshold. Thus, the shapes of the bifurcations, their locations, and their evolutions with the noise intensity level, in the vicinity of the critical point, are completely defined as 
well as the analytical expressions for the bistability region and Maxwell point in the subcritical case. Features are studied and lead to, for instance, the proposition of a criterion for the determination of the state phase change point in the supercritical case or the evidence of total elimination of the hysteresis cycle by sufficient additive noise level in the subcritical case. Hysteresis cycle suppression by noise has been suggested theoretically in different models of subcritical bifurcations in extended systems [19,21] and observed experimentally in electronic circuits and surface waves generated by vertically vibrating a layer of fluid [22]. Numerical simulations are carried out for prototype models of pattern formation based on stochastic super- and subcritical Swift-Hohenberg equations, which show quite good agreement with our theoretical predictions.

The paper is organized as follows. In Sec. II, we present several examples of numerical observations of the effects of additive noise on spatial bifurcations. In Sec. III, we give the amplitude equations of two stochastic supercritical bifurcations (one corresponding to a cubic equation and one associated to a quintic equation) and of a stochastic sub-critical bifurcation (cubic-quintic amplitude equation). Sections IV to VI are devoted to the derivation of analytical expression of the most probable amplitude fully describing the stochastic bifurcations of the previously mentioned amplitude equations.

\section{EVIDENCE OF NOISE EFFECTS ON BIFURCATIONS}

Let us consider a one-dimensional extended system that exhibits a spatial bifurcation, either supercritical or subcritical, described by

$$
\partial_{t} \vec{u}=\vec{f}\left(\vec{u}, \partial_{x},\{\lambda\}\right)+\sqrt{\eta^{\prime}} \vec{\zeta}(x, t),
$$

where $\vec{u}(x, t)$ is a field that accounts for the system under study, $\vec{f}$ is the vector field, $\{\lambda\}$ a set of parameters that characterize the system under study, $\eta^{\prime}$ is the noise intensity that satisfies for equilibrium system to be proportional to, e.g., the temperature, and $\vec{\zeta}(x, t)$ is a white Gaussian noise with zero mean value and correlation

$$
\left\langle\zeta^{i}(x, t) \zeta^{j}\left(x^{\prime}, t^{\prime}\right)\right\rangle=C^{i j} \delta\left(t-t^{\prime}\right) \delta\left(x-x^{\prime}\right),
$$

where $C^{i j}$ is the correlation matrix which is symmetric and semidefinite positive. For $\eta^{\prime}=0$ the above model is usually denominated deterministic system. Assuming that the above system has a stable homogeneous uniform state $\vec{u}_{o}$, that is, $\vec{f}\left(\vec{u}_{o}, \partial_{x},\{\lambda\}\right)=0$, and the set of eigenvalues associated to linear operator around $\vec{u}_{o}$ has negative real part. For a critical value of the parameters $\left\{\lambda_{c}\right\}$, it exhibits a linear stationary spatial instability as a perturbation of the deterministic system,

$$
\vec{u}(x, t)=\vec{u}_{o}+e^{\sigma(k) t+i k x} \hat{u},
$$

where $\sigma(k)$ is the linear growth rate and $\hat{u}$ an arbitrary constant vector. The linear theory tells that, if $\sigma(k)<0$, then the perturbation of the uniform state $\vec{u}_{o}$ decreases and disappears. The growth rate at the spatial bifurcation has a global maximum for the non-null wave number at critical $k=$ $k_{c}$, as depicted in Fig. 1. Above (below) the bifurcation point and close to the critical wave number, the growth rate curve is larger (smaller) than the critical growth rate at the bifurcation transition (cf. Fig. 1). Hence, below the spatial bifurcation

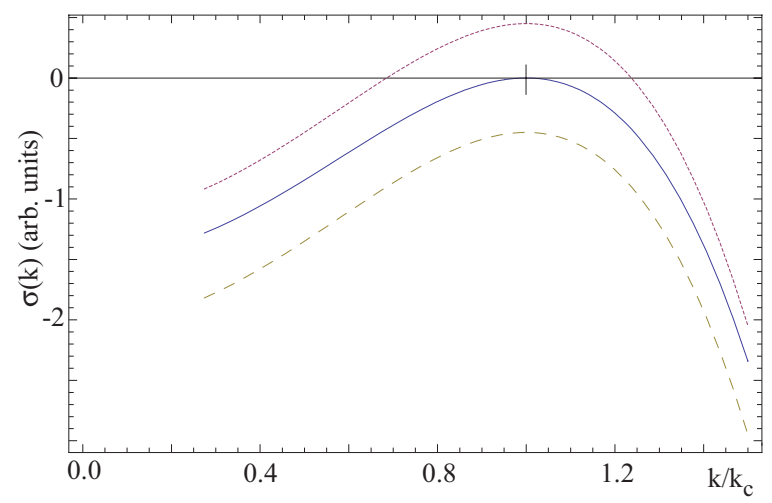

FIG. 1. (Color online) Growth rate as function of wave number $k$. Dashed, continuous, and dotted curves denote growth rate below, at, and above the spatial bifurcation. $k_{c}$ is the critical wave number.

when noise is considered, all spatial modes are excited and the slowest mode rules the dynamics. The dynamical behavior of the system is characterized by noise-supported patterns (cf. Fig. 2). This phenomenon is well known as a pattern precursor [23]. Figure 2 shows the typical precursor observed below the spatial bifurcation for a prototype model of pattern formation, the Swift-Hohenberg model [cf. Eq. (11)], and a typical profile registered at a given time (inset of Fig. 2). A direct consequence is that the stochastic bifurcation is no more characterized by a discontinuous or nondifferentiable transition between the uniform and the structured pattern as it is the case for the deterministic transitions (cf. insets of Fig. 3).

Figure 3 shows the time-average modulus of the Fourier transform $S(k)$ of the field $\vec{u}$, also called the structure function, for the supercritical [Fig. 3(a)] and subcritical [Fig. 3(b)] deterministic Swift-Hohenberg equations [19]. Their insets depict the corresponding deterministic bifurcations and the respective value of the bifurcation parameter, where the structure function is calculated. It is noteworthy that even below the deterministic threshold (before the hysteresis region

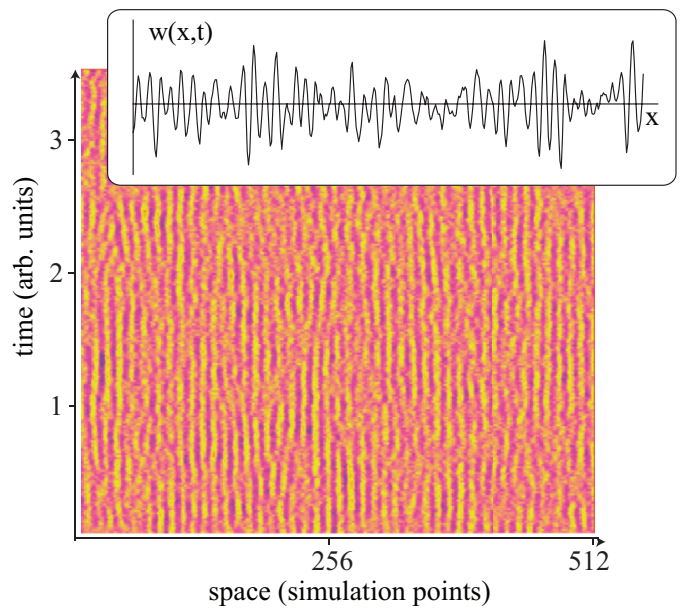

FIG. 2. (Color online) Pattern precursors: spatiotemporal diagram observed in the Swift-Hohenberg equation (11) below the spatial bifurcation. The vertical and horizontal axis stand for time and space, respectively. Inset: the instantaneous profile of the Swift-Hohenberg equation. 


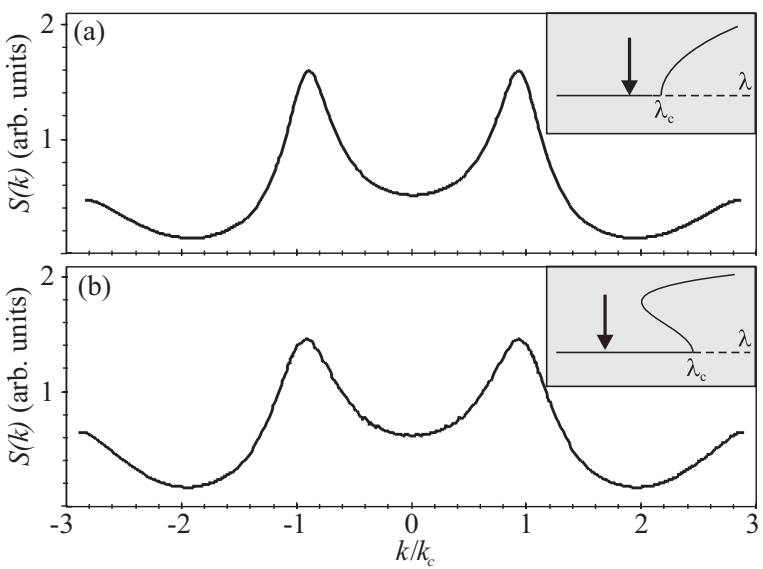

FIG. 3. Stationary structure function $S(k)$, obtained from the supercritical (a) and subcritical (b) Swift-Hohenberg model below the bifurcation point. Insets: The arrows indicate the corresponding values of the control parameter $\lambda$ with respect to this critical value $\lambda_{c} . k_{c}$ is the slowest mode.

for the subcritical case), the structure function gives the value of the coming critical wave number and its associated amplitude. Hence, if these functions have a maximum at a given wave number, then this mode is the most recurrent observed mode (see Figs. 2 and 3).

In the presence of noise, to describe the whole shape of the noisy bifurcations in a unified manner, we have to consider, even for weak values of the control parameter, the amplitude of this most recurrent mode since the evolution of the amplitude of the spatial critical mode at threshold represents the bifurcation diagram in the vicinity of the deterministic threshold.

\section{NOISY AMPLITUDE EQUATIONS}

To describe the dynamical behavior of a system in a unified description close to the instability, we always can consider the ansatz $[3,12]$

$$
\begin{aligned}
\vec{u}(x, t)= & \vec{u}_{o}+\varepsilon^{\gamma} C(T=\varepsilon t, X=\sqrt{\varepsilon} x) e^{i k_{c} x} \hat{u}_{k} \\
& +\varepsilon^{\gamma} C^{*}(T, X) e^{-i k_{c} x} \hat{u}_{k}^{*}+\text { h.o.t. },
\end{aligned}
$$

where $C$ is the amplitude of the critical mode; the star symbol $(*)$ denotes the complex conjugate; $\varepsilon$ is the bifurcation parameter and is proportional to $\lambda-\lambda_{c}$, where $\lambda$ is the control parameter; and $\gamma$ exponent controls the scaling of the amplitude $C$, which is determined by the first dominant nonlinearity. $\hat{u}_{k}$ is the eigenvector associated to the critical mode. $\{T, X\}$ are, respectively, slow time and spatial variables and the high-order term (h.o.t.) are a series in the amplitude $C$. To understand this ansatz, we can recall that, above the threshold, unstable modes can be written as a wave packet,

$$
\int a_{k^{\prime}} e^{i k_{c} x+i \sqrt{\varepsilon} k^{\prime} x} d k^{\prime}=\underbrace{\left(\int a_{k^{\prime}} e^{i \sqrt{\varepsilon} k^{\prime} x} d k^{\prime}\right)}_{C(X=\sqrt{\varepsilon} x)} e^{i k_{c} x} .
$$

Hence, the amplitude $C$ describes the dynamic of whole unstable modes close to the bifurcation. $L$ is the transverse size of the system in consideration. On the basis of the theory of normal forms $[24,25]$ and amplitude equations $[3,12]$ the amplitude $C$ satisfies

$$
\partial_{T} C=\varepsilon C+b|C|^{2} C+h|C|^{4} C+\sqrt{\eta} \varsigma(T)+\text { h.o.t. }
$$

where $b$ and $h$ are the nonlinear real coefficients, h.o.t. represent the high-order terms, and

$$
\varsigma(T) \equiv \frac{k_{c}}{2 \pi} \int_{0}^{2 \pi / k_{c}} d x \vec{\zeta}(x, t) \cdot \hat{u}_{k}\left(\cos k_{c} x+i \sin k_{c} x\right)
$$

is a stochastic field, formed by a pair of Gaussian white noise with zero mean value and correlation $\left\langle\varsigma(T) \varsigma\left(T^{\prime}\right)\right\rangle=0$, $\left\langle\varsigma^{*}(T) \varsigma^{*}\left(T^{\prime}\right)\right\rangle=0$ and $\left\langle\varsigma(T) \varsigma^{*}\left(T^{\prime}\right)\right\rangle=\delta\left(T-T^{\prime}\right) . \eta$ is the intensity of noise level for the amplitude equation (6), which is related to the initial noise level $\eta^{\prime}$ in Eq. (1) by $\eta=\eta^{\prime} k_{c} / 2 \pi$.

\section{A. Subcritical bifurcation}

In the case of positive $b$, the amplitude equation (6) describes a subcritical bifurcation. However, in order that the amplitude equation accounts qualitatively and quantitatively for the dynamics, one must consider $b$ small, and the specific following scalings $\gamma=1 / 4, \partial_{T} \sim \varepsilon, b / h^{1 / 2} \sim \varepsilon^{1 / 4}, \varepsilon \ll 1$, $C=A / \sqrt[4]{|h|}$, and $h<0$, the amplitude equation then reads

$$
\partial_{T} A=\varepsilon A+\frac{b}{\sqrt{h}}|A|^{2} A-|A|^{4} C+\sqrt{\eta \sqrt{h}} \varsigma(T) .
$$

It is important to note that to derive the above equation, we need to consider a codimension two point, that is, it is necessary to fix two conditions in the parameter space, namely the linear and cubic coefficients have to be small and related by the above scaling. However, the subcritical bifurcation is codimension 1 . This contradiction is a consequence of the analytical equation (8) which is only valid for small cubic coefficients (see discussion in Ref. [26]).

\section{B. Supercritical bifurcations}

\section{Quintic supercritical bifurcation}

A particular case of the above Eq. (8) is the codimension two point $b=0$, that is,

$$
\partial_{T} A=\varepsilon A-|A|^{4} C+\sqrt{\eta \sqrt{h}} \varsigma(T) .
$$

This amplitude equation (9) accounts for a supercritical bifurcation. We term this bifurcation quintic supercritical bifurcation.

\section{Cubic supercritical bifurcation}

For negative $b$, Eq. (6) accounts for a cubic supercritical spatial bifurcation. Using the scaling $\gamma=1 / 2, \partial_{T} \sim \varepsilon, \varepsilon \ll 1$, and $C=A / \sqrt{|b|}$, it then can be approached by

$$
\partial_{T} A=\varepsilon A-|A|^{2} A+\sqrt{b \eta} \varsigma(T) .
$$

\section{Example of amplitude equation derivation for a supercritical bifurcation}

To illustrate the procedure of deduction of the amplitude equation we consider the stochastic supercritical 
Swift-Hohenberg model [24]

$$
\partial_{t} w=\lambda w-w^{3}-\left(\partial_{x x}+q^{2}\right)^{2} w+\sqrt{\sigma_{0}} \zeta(x, t),
$$

where $w(x, t)$ is a real field, $\lambda$ is the bifurcation parameter, $q$ is the wave number of periodical solutions, $\zeta(x, t)$ is a scalar Gaussian white noise with zero mean value and correlation $\left\langle\zeta(x, t) \zeta\left(x^{\prime}, t^{\prime}\right)\right\rangle=\delta\left(x-x^{\prime}\right) \delta\left(t-t^{\prime}\right)$, and $\sigma_{0}$ accounts for the intensity of noise level.

A trivial uniform stationary state of the deterministic model (11) is $w(x, t)=0$. This state is stable for $\lambda<0$ and exhibits a supercritical spatial instability for $\lambda_{c}=0$, which gives rise to the appearance of a pattern with wave number $q$ for $\lambda>0$. Hence, the critical wave number is $k_{c}=q$. We consider the small bifurcation parameter $\varepsilon$ such that $\varepsilon=\lambda-\lambda_{c} \ll 1$ and small intensity of noise level $\sqrt{\sigma_{0}} \sim \epsilon$. The system size is $L$ and, for the sake of simplicity, we consider the periodic boundary conditions $w(x=-L / 2, t)=w(x=L / 2, t)$. Note that all the following results presented in the article assume that $L$ is sufficiently large, i.e., in practical terms $L$ is considered infinite. To grasp the dynamics of Eq. (11) we introduce the following ansatz of the single critical mode:

$$
w=\frac{A(T)}{\sqrt{3}} e^{i q x}+\frac{A^{*}(T)}{\sqrt{3}} e^{-i q x}+U\left(A, A^{*}, x\right),
$$

where $q \approx 2 \pi m / L$ and $m$ is a given integer. For wide-enough system size $L, A(T)$ is the space-independent amplitude of the critical mode $q$ and $U\left(A, A^{*}, x\right)$ is a small correction function including high-order terms in $A, A^{*}$. Note that this nonlinear function $U\left(A, A^{*}, x\right)$ can be written as a power series in the amplitude $A$ [25]. Because the spatial bifurcation of model (11) is supercritical, the amplitude $A$, slow time $T=\varepsilon t$, and $U\left(A, A^{*}, x\right)$ are order of $\varepsilon^{1 / 2}, \varepsilon$, and $\varepsilon^{3}$, respectively. Introducing the ansatz (12) in Eq. (11), we obtain at order $\varepsilon^{3}$

$$
\begin{aligned}
\left(\partial_{x x}+q^{2}\right)^{2} U= & \left\{-\partial_{T} A+\varepsilon A-|A|^{2} A\right\} \frac{e^{i q x}}{\sqrt{3}} \\
& -\frac{A^{3}}{3 \sqrt{3}} e^{i 3 q x}+\sqrt{\sigma_{0}} \zeta(x, t)+\text { c.c. },
\end{aligned}
$$

where c.c. denotes complex conjugate. The linear operator $\left(\partial_{x x}+q^{2}\right)^{2}$ is a self-adjoint with the inner product

$$
\langle f(x, t) \mid g(x, t)\rangle=\frac{q}{2 \pi n} \int_{0}^{2 \pi n / q} d x f(x, t) g(x, t)^{*},
$$

where $n$ is an arbitrary integer. The linear operator is not invertible because $\left(\partial_{x x}+q^{2}\right)^{2} e^{ \pm i q x}=0$, that is, $e^{ \pm i q x}$ are elements of the kernel of operator $\left(\partial_{x x}+q^{2}\right)^{2}$. In order to have a solution for $U$ in Eq. (13), we multiply the right-hand side of Eq. (13) by $q e^{-i q x} / 2 \pi n$, integrate in the whole domain, and impose that it is equal to zero (Fredholm alternative or solvability condition [12]). Hence, we obtain the amplitude equation

$$
\partial_{T} A=\varepsilon A-|A|^{2} A+\sqrt{\eta} \varsigma(t),
$$

where $\eta=3 \sigma_{0}$ and

$$
\zeta(t) \equiv \frac{q}{2 \pi n} \int_{0}^{2 \pi n / q} \zeta(x, t) e^{-i q x} d x,
$$

where $\zeta$ is a Gaussian noise, because it is a combination of large number of independent Gaussian noises (central limit theorem). Hence, we need to characterize the mean value and their respective correlation functions using the features of the stochastic field $\zeta(x, t)$. The mean value satisfies

$$
\begin{aligned}
\langle\varsigma(t)\rangle & \equiv\left\langle\frac{q}{2 \pi n} \int_{0}^{2 \pi n / q} \zeta(x, t) e^{-i q x} d x\right\rangle_{\zeta}, \\
& =\frac{q}{2 \pi n} \int_{0}^{2 \pi n / q}\langle\zeta(x, t)\rangle_{\zeta} e^{-i q x} d x, \\
& =0,
\end{aligned}
$$

because the initial noise has a zero mean value $\left(\langle\zeta(x, t)\rangle_{\zeta}=0\right)$ and its correlation $\left\langle\zeta(x, t) \zeta\left(x^{\prime}, t^{\prime}\right)\right\rangle_{\zeta}=\delta\left(x-x^{\prime}\right) \delta\left(t-t^{\prime}\right)$. The correlation functions read

$$
\begin{aligned}
& \langle\varsigma(t) \varsigma(t)\rangle \\
& \quad=\left(\frac{q}{2 \pi n}\right)^{2} \iint_{0}^{2 \pi n / q} d x d x^{\prime}\left\langle\zeta(x, t) \zeta\left(x^{\prime}, t^{\prime}\right)\right\rangle_{\zeta} e^{-i q\left(x+x^{\prime}\right)}, \\
& \quad=\left(\frac{q}{2 \pi n}\right)^{2} \iint_{0}^{2 \pi n / q} d x d x^{\prime} \delta\left(x-x^{\prime}\right) \delta\left(t-t^{\prime}\right) e^{-i q\left(x+x^{\prime}\right)}, \\
& \quad=\delta\left(t-t^{\prime}\right)\left(\frac{q}{2 \pi n}\right)^{2} \int_{0}^{2 \pi n / q} e^{-i q 2 x} d x, \\
& \quad=0
\end{aligned}
$$

and

$$
\begin{aligned}
& \left\langle\varsigma(t) \zeta^{*}(t)\right\rangle \\
& =\left(\frac{q}{2 \pi n}\right)^{2} \iint_{0}^{2 \pi n / q} d x d x^{\prime}\left\langle\zeta(x, t) \zeta^{*}\left(x^{\prime}, t^{\prime}\right)\right\rangle_{\zeta} e^{-i q\left(x-x^{\prime}\right)}, \\
& =\left(\frac{q}{2 \pi n}\right)^{2} \iint_{0}^{2 \pi n / q} d x d x^{\prime} \delta\left(x-x^{\prime}\right) \delta\left(t-t^{\prime}\right) e^{-i q\left(x-x^{\prime}\right)}, \\
& =\delta\left(T-T^{\prime}\right)\left(\frac{q}{2 \pi n}\right)^{2} \int_{0}^{2 \pi n / q} d x, \\
& =\left(\frac{q}{2 \pi n}\right)^{2} \delta\left(t-t^{\prime}\right) .
\end{aligned}
$$

Therefore, the effective intensity of noise is $3 q \sigma_{0} / 2 \pi n$. The intensity of the noise level is of order $\epsilon^{2}$, meaning that all the terms on the right-hand side of Eq. (14) are of order $\epsilon$ and the temporal variation of the amplitude $A$ is of order $\epsilon$. This is consistent with the scaling under consideration. Using the solvability condition, at dominant order the correction function $U$ takes the form

$$
U=-\frac{A^{3}}{\left(8 q^{2}\right)^{2} 3 \sqrt{3}} e^{i 3 q x}-\frac{A^{* 3}}{\left(8 q^{2}\right)^{2} 3 \sqrt{3}} e^{-i 3 q x} .
$$

In the next sections we discuss the effect of the noise in the different bifurcations.

\section{CUBIC SUPERCRITICAL SPATIAL BIFURCATION}

Let us consider a noisy supercritical bifurcation, which is described by model (10). To this Langevin equation is associated the following Fokker-Planck equation for the distribution probability $P\left(A, A^{*} ; T\right)$ of the critical mode amplitude 


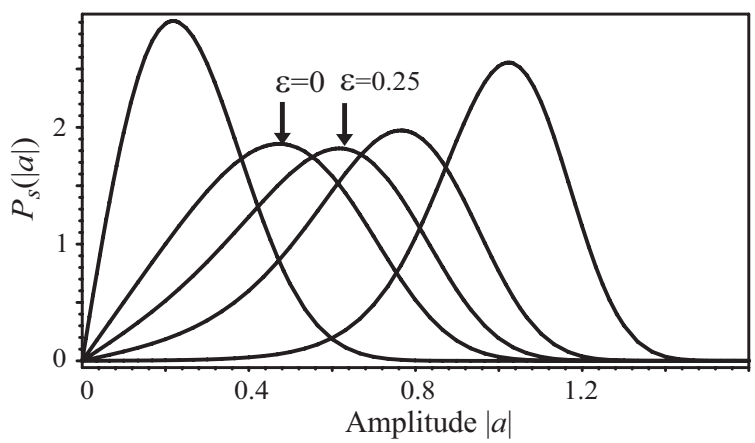

FIG. 4. Stationary probability distribution of the modulus $|A|$, from formula (17), for different bifurcation parameter values. From left to right: $\varepsilon=-1,0,0.25,0.5,1 . \eta=0.1$.

$A[27,28]$ :

$$
\begin{aligned}
\partial_{T} P= & \partial_{A}\left\{-\varepsilon A+|A|^{2} A+\frac{\eta}{2} \partial_{A^{*}}\right\} P \\
& +\partial_{A^{*}}\left\{-\varepsilon A^{*}+|A|^{2} A^{*}+\frac{\eta}{2} \partial_{A}\right\} P .
\end{aligned}
$$

Its stationary value has the form

$$
P_{s}\left(A, A^{*}\right)=\tilde{Q}_{\text {sup }}(\varepsilon, \eta) e^{\frac{\varepsilon|A|^{2}-\frac{|A|^{4}}{2}}{\eta}},
$$

where $\tilde{Q}_{\text {sup }}(\varepsilon, \eta)$ is the normalization factor. Introducing the polar representation $\left(A=|A| e^{\phi}\right.$, where $\{|A|, \phi\}$ stand for the modulus and phase, respectively) and integrating over the phase one obtains the stationary probability density of the modulus of the critical mode amplitude $A$ (generalized Rayleigh distribution [29]),

$$
P_{s}(|A|)=Q_{\text {sup }}(\varepsilon, \eta)|A| e^{\frac{\varepsilon|A|^{2}-\mid \frac{|A|^{4}}{2}}{\eta}},
$$

where $Q_{\text {sup }}(\varepsilon, \eta)$ is the appropriate normalization factor. This function is plotted in Fig. 4 for various values of the control parameter $\varepsilon$. The maximum of the generalized Rayleigh distribution occurs at the most probable value [20],

$$
\left|a_{\max }\right|=\sqrt{\frac{\varepsilon+\sqrt{\varepsilon^{2}+2 \eta}}{2}} .
$$

This is an exact expression derived from the generalized Rayleigh distribution, expression (17). When $\varepsilon<0$ the most probable value is close to zero $\left(\left|a_{\max }\right| \approx-\eta / 2 \varepsilon,|\varepsilon| \gg 1\right)$ and the width of the stationary probability distribution decreases when the bifurcation parameter is diminished. For $\varepsilon>0$, the most probable is close to $\sqrt{\varepsilon+\eta / 2 \varepsilon}(|\varepsilon| \gg 1)$ and the width of the stationary probability distribution decreases when the bifurcation parameter is increased. This phenomenon is a consequence of the increase of the Lyapunov exponents when departing from the deterministic bifurcation point $\varepsilon=0$ [16].

Starting from the probability distribution of the modulus of $A$, formula (17), two natural quantities can be used to draw a noisy bifurcation diagram: the nonlinear mean value $\langle|a|\rangle_{\text {nonlinear }}$ and the most probable value $\left|a_{\max }\right|$. As it can be seen in Fig. 5, for large $\varepsilon$ each of these curves matches the deterministic bifurcation curve. However, near the deterministic bifurcation point, fluctuations induce a

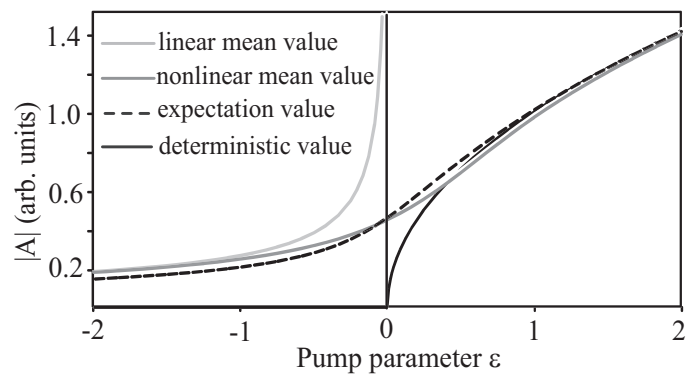

FIG. 5. Bifurcation diagrams of the amplitude of the critical mode obtained for different resolutions of Eq. (14). Namely the deterministic value $A$, the expectation value $\left|a_{\max }\right|$, the linear and nonlinear mean values $\langle|a|\rangle_{\text {linear }}$ and $\langle|a|\rangle_{\text {nonlinear }}$, respectively (see text for value definitions).

smoothing of the curves. If we neglect the nonlinear term in the Langevin equation (10), one can compute the linear mean value of the amplitude modulus $\langle|a|\rangle_{\text {linear }}=\sqrt{-\pi \eta / 4 \varepsilon}$. Note that this linear mean value, used in most of the related works [30,31], is well defined for $\varepsilon<0$ but diverges at the bifurcation point. The $\langle|a|\rangle_{\text {linear }}$ and $\langle|a|\rangle_{\text {nonlinear }}$ curves are quite similar for large negative $\varepsilon$; however, they differ close to the bifurcation. In the following, we will use the most probable value as reference quantity, since it is the most relevant and useful quantity to describe experimental observations [20,29], given the limitation of the finite duration of data recording. It is important to note that for such a finite duration time, it is simpler to identify the most probable value, as it is the most observed value, in comparison with the mean value, which is more sensitive to accessible spatial sampling. Therefore, the convergence of the most probable value is much more efficient than that of the mean value.

To emphasize the universality of the above analysis, Fig. 6 shows the comparison between numerical data obtained from the subcritical Swift-Hohenberg model (11) and the corresponding analytical expression (18). We can see that the expression (18) perfectly fits and reproduces the noisy numerical bifurcation. Thus, this anaytical expression includes all the information concerning the shape of the bifurcation, its location, and its evolution with the noise intensity level.

Note that close to the bifurcation point, $P\left(\left|A_{\max }\right|\right)$ exhibits a minimum (see Fig. 4) corresponding to dynamics characterized by large fluctuations. This feature indicates a phase-state

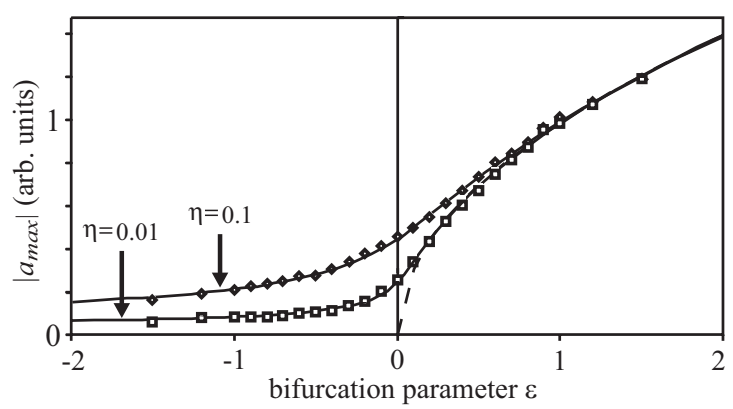

FIG. 6. Bifurcation diagram of supercritical stochastic SwiftHohenberg equation (31) for two different noise intensities. Continuous curves show the fits using the expression (18). The dashed curve is the corresponding bifurcation without noise. 
change in the system and, thus, the location of the phase transition between the two states. This minimum, occuring at $\varepsilon_{\min }$, does not coincide to the deterministic threshold ( $\varepsilon=0)$. To determine $\varepsilon_{\min }$, we first need to calculate the factor $Q_{\text {sup }}(\varepsilon, \eta)$. Introducing the change of variable $x=$ $\left(|A|^{2}-\varepsilon\right) / \sqrt{2 \eta}$, and using the normalization condition

$$
\sqrt{2 \eta} Q_{\text {sup }}(\varepsilon, \eta) e^{\frac{\varepsilon^{2}}{2 \eta}} \int_{-\frac{\varepsilon}{\sqrt{2 \eta}}}^{\infty} e^{-x^{2}} d x=1,
$$

the normalization factor reads

$$
Q_{\text {sup }}(\varepsilon, \eta)=\frac{2 \sqrt{2}}{\sqrt{\pi \eta}} \frac{e^{-\frac{\varepsilon^{2}}{2 \eta}}}{\operatorname{erf} c\left(-\frac{\varepsilon}{\sqrt{2 \eta}}\right)},
$$

where $\operatorname{erf} c(z)=1-\operatorname{erf}(z)$ and $\operatorname{erf}(z)$ is the error function. Putting (18) and (20) into (17), we obtain the whole expression of the probability density maximum,

$$
P\left(\left|a_{\max }\right|\right)=\frac{2^{\frac{5}{4}}}{\eta^{\frac{1}{4}} \sqrt{\pi}} \frac{e^{-\alpha^{2}-\frac{1}{4}}}{\operatorname{erfc}(-\alpha)} \sqrt{\alpha+\sqrt{\alpha^{2}+1}} e^{\frac{1}{2}\left(\alpha^{2}+\alpha \sqrt{\alpha^{2}+1}\right)}
$$

with $\alpha=\varepsilon / \sqrt{2 \eta}$. This function reaches a minimum for $\alpha_{\min }=0.389$. Thus, the bifurcation parameter value $\left(\varepsilon_{\min }(\eta)\right)$, corresponding to the minimum of the most probable value, is

$$
\varepsilon_{\min }(\eta) \approx 0.55 \sqrt{\eta}
$$

It locates the phase-state change point along the bifurcation diagram. Note that a similar expression can be obtained by calculating the minimum value of the Lyapunov exponent as a function of the bifurcation parameter [16]. Since the system is noisy, one can no longer speak about "threshold," which is associated only with deterministic systems. We have to consider bifurcations in the sense of phase-transition diagrams such as second-order transitions in material sciences. In other words, we have to deal with phase transitions in the sense of Landau [2] for noisy systems. The shift between the minimum of $P\left(\left|a_{\max }\right|\right)$ associated with the control parameter value $\lambda_{\text {min }}$ and the deterministic threshold corresponding to $\lambda_{c}$ is

$$
\lambda_{\text {min }}=\lambda_{c}+0.55 \sqrt{\eta} .
$$

Experimentally, it is quite easy to build the probability density distribution. Then, with the help of the fitted values obtained from the relation (18), $\varepsilon_{\min }$ can be determined and, thus, the noise level intensity $\eta$ estimated.

As shown in Refs. [20,29], the analytical expression of $\left|a_{\max }\right|$ perfectly fits the experimental noisy bifurcation obtained in $1 \mathrm{D}$ optical patterns observed in a Kerr-like (liquid crystal slice) feedback system [Fig. (7)] and a fluidized quasi-onedimensional shallow granular bed [29]. In the optical setup, the roll pattern appears above a critical input laser intensity $I_{c}$ through a supercritical bifurcation [32]. Thermal fluctuations of the liquid crystal molecules around their equilibrium position transform the shape of the standard bifurcation into a noisy one. Below the deterministic threshold, the ouput signal is characterized by noisy precursors, without fixed spatial phase but with a clear dominant mode with non-null amplitude [23]. Above threshold, the selective spatial amplification is more effective and the system reaches a stationary pattern. The correponding bifurcation diagram is plotted in Fig. 7. It

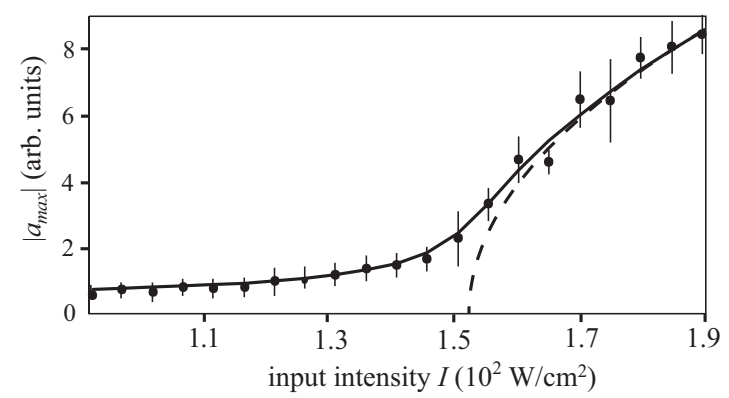

FIG. 7. Experimental bifurcation diagram for the optical feedback system. The continuous curve is the analytical expression and dots are experimental values. Error bars correspond to the full width at half maximum of the experimental probability distribution.

evidences the good agreement between the analytical curve and the experimental data of $\left|a_{\max }\right|$. The values of $\eta$ and $I_{c}$ obtained from the fit are 0.01 and $152 \mathrm{~W} / \mathrm{cm}^{2}$. They are in good agreement with the previous determination method [23].

\section{QUINTIC SUPERCRITICAL BIFURCATION}

An another example of supercritical bifurcation is the quintic supercritical, which is usually reached in parametrically driven systems [33]. A classical example of this bifurcation is a vertically driven chain of pendula [34]. Figure 8 shows the structure factor obtain in the vertically driven pendula chain when the amplitude of the forcing is close to the half of damping. Hence, this system exhibits a precursor and the amplitude of the standing wave increases with the power law 1/4. The inset of Fig. 8 depicts the associated deterministic bifurcation diagram. Experimentally, the verification of this type of bifurcation has been achieved in parametric surface waves in mercury [35]. Close to the spatial bifurcation the amplitude equation that describes this bifurcation is given by Eq. (9).

For the sake of simplicity, let us introduce $\sigma \equiv \eta \sqrt{h}$. Associated to the above stochastic equation one has the following Fokker-Planck equation for the distribution probability

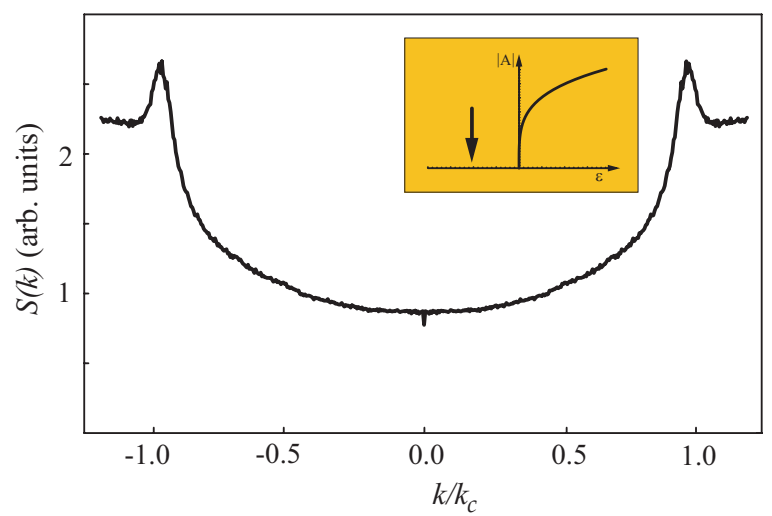

FIG. 8. (Color online) Stationary structure function $S(k)$, obtained from a vertically driven pendula chaim. Inset: The deterministic bifurcation diagram and the arrow indicate the corresponding value of the control parameter. 


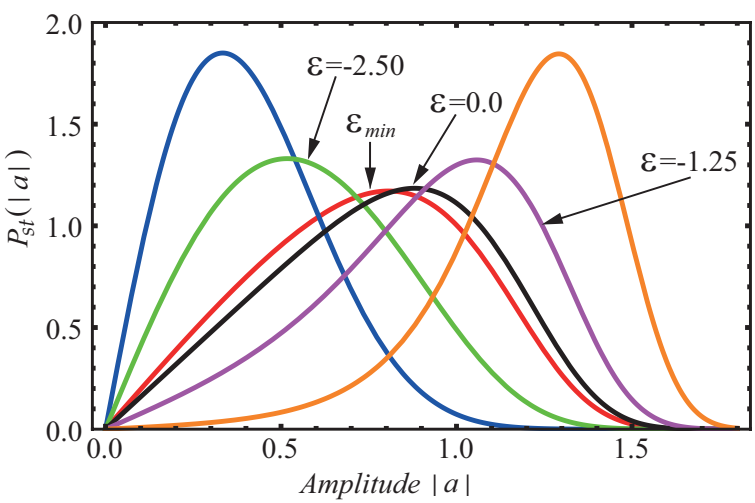

FIG. 9. (Color online) Stationary probability distribution for different values of the bifurcation parameter $\varepsilon$ and $\sigma_{0}=1.4$. From left to right: $\varepsilon=-6.26 ;-2.50 ; \varepsilon_{\min }=-0.35,0.0,1.25,3.75$.

$P\left(A, A^{*} ; T\right)[27,28]$,

$$
\begin{aligned}
\partial_{T} P= & \partial_{A}\left\{-\varepsilon A+|A|^{4} A+\frac{\sigma}{2} \partial_{A^{*}}\right\} P \\
& +\partial_{A^{*}}\left\{-\varepsilon A^{*}+|A|^{4} A^{*}+\frac{\sigma}{2} \partial_{A}\right\} P .
\end{aligned}
$$

The stationary distribution probability of the modulus of $A$ has the form

$$
P_{S}(|A|)=Q_{\text {sup }}(\varepsilon, \sigma)|A| e^{\frac{\varepsilon|A|^{2}-\frac{|A|^{6}}{2}}{\sigma}},
$$

where $Q_{\text {sup }}(\varepsilon, \sigma)$ is the normalization factor. The stationary probability is shown in Fig. 9 for various values of the control parameter $\varepsilon$. The respective maximum of the stationary distribution probability occurs at the most probable value (25)

$$
\left|a_{\max }\right|=\sigma^{1 / 6} \sqrt{\frac{\beta}{\Omega}+\frac{\Omega}{3}},
$$

where $\Omega \equiv(3 / 4)^{1 / 3}\left(9+\sqrt{3} \sqrt{27-16 \beta^{3}}\right)^{1 / 3}$ and $\beta=\varepsilon / \sigma^{2 / 3}$. When $\varepsilon<0$ the most probable amplitude value is close to zero $(\sqrt{-\sigma / 2 \varepsilon},|\varepsilon| \gg \sqrt{\sigma})$ and the width of the stationary probability distribution decreases when the bifurcation parameter is diminished. For $\varepsilon>0,\left|a_{\max }\right|$ is close to $\sqrt[4]{\epsilon}+\sigma / 4 \varepsilon^{3 / 2}(|\varepsilon| \gg$ $\sqrt{\sigma}$ ) and the width of the stationary probability distribution decreases when the bifurcation parameter is increased. This

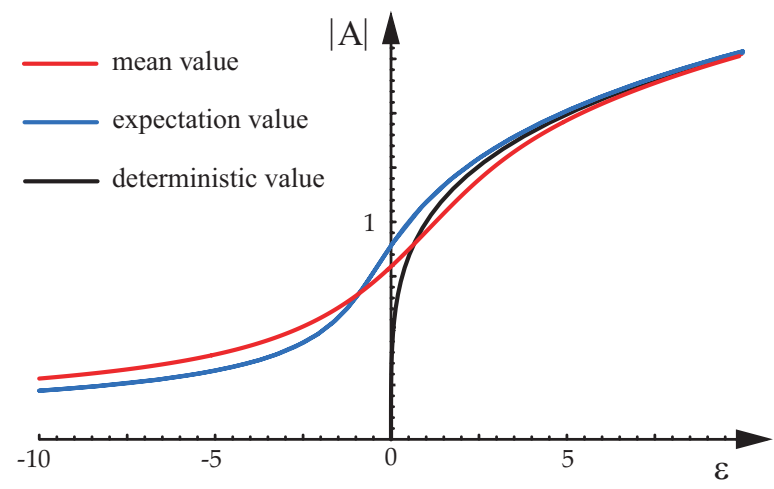

FIG. 10. (Color online) Bifurcation diagrams obtained from the different calculs of the amplitude (see text for definitions).

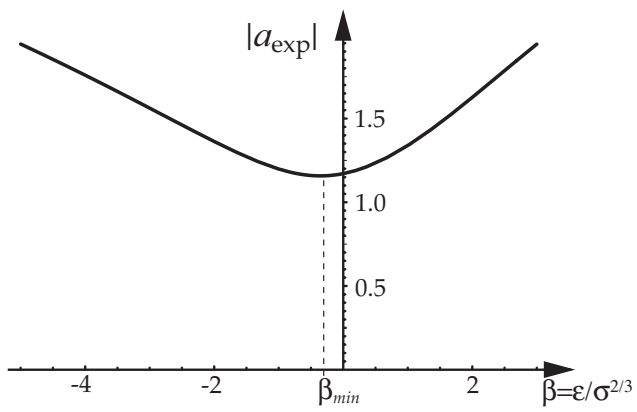

FIG. 11. Evolution of the most probable value $\left|a_{\max }\right|$ versus the bifurcation parameter $\beta=\varepsilon / \sigma^{2 / 3}$. $\beta_{\min }$ stands for the minimum of the curve of $\left|a_{\max }\right|$ and then indicates the phase transition in the system.

is again the consequence of the increase of the Lyapunov exponents when departing from the bifurcation point (Sec. IV). Starting from the probability distribution again, the natural quantity used to draw the noisy bifurcation diagram is the most probable amplitude $\left|a_{\max }\right|$. As can be seen in Fig. 10, for large $\varepsilon,\left|a_{\max }\right|$ matches the deterministic bifurcation. However, near the deterministic threshold, fluctuations induce a smoothing of the curve near the deterministic threshold. Note that close to the bifurcation point corresponding to the phase transition, $P\left(\left|a_{\max }\right|\right)$ also exhibits a minimum (see Fig. 11). The dynamics is characterized by large fluctuations. This minimum $\varepsilon_{\min }$ does not coincide with the deterministic threshold $\left(\varepsilon_{\min }=0\right)$. Numerically, we have determined the expression of the minimum and it reads

$$
\varepsilon_{\min }(\sigma) \approx-0.3537 \sigma^{2 / 3} \text {. }
$$

Hence, in a similar manner as for the cubic supercritical bifurcation, the localization of the phase transition within the noisy bifurcation is given by

$$
\lambda_{\min }=\lambda_{c}-0.3537 \sigma^{2 / 3} .
$$

The reason why $\varepsilon_{\min }$ is negative is related to the fact that the modulus of Lyapunov exponents are larger than the respective Lyapunov exponents of the cubic supercritical bifurcation.

\section{SUBCRITICAL SPATIAL BIFURCATION}

Several physical systems leave their equilibrium via a discontinuous spatial transition, that is, an infinitesimal increase of the control parameter leads to a jump of the order parameter $[3,19]$. Close to a spatial subcritical bifurcation the system is described by Eq. (8); rescaling the time, this equation then reads

$$
\partial_{T} A=\varepsilon^{\prime} A+|A|^{2} A-|A|^{4} C+\sqrt{\eta^{\prime}} \varsigma(T) .
$$

In a similar manner to that of the supercritical case, we can obtain the respective stationary probability distribution of the modulus of $A$,

$$
P_{S}(|A|)=Q_{\text {sub }}\left(\varepsilon^{\prime}, \eta^{\prime}\right)|A| e^{\frac{\varepsilon^{\prime} \frac{|A|^{2}}{2}+\frac{|A|^{4}}{4}-\frac{|A|^{6}}{6}}{\eta^{\prime}}} .
$$

Two different scenarios appear depending on the level of noise. For small $\eta^{\prime}$ and an interval of $\varepsilon\left(\varepsilon_{\text {up }}<\varepsilon<\varepsilon_{\text {down }}\right)$, the distribution function $P_{s}(|A|)$ possess two distinct maxima [see Fig. 12(b)], which reveals the presence of bistability. Above 


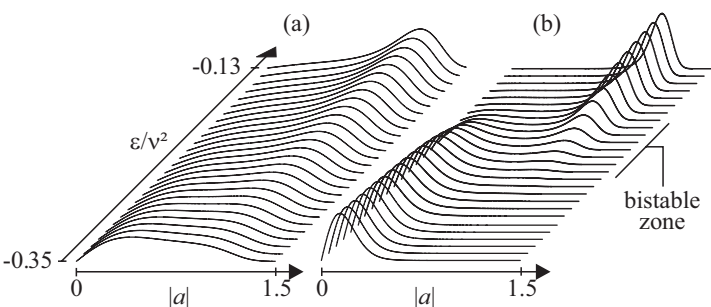

FIG. 12. Stationary probability distribution $P_{s}(|a|)$ for different values of the bifurcation parameter $\varepsilon$ and $\eta^{\prime}$. (a) $\eta^{\prime}=0.08>\eta_{c}^{\prime}$; (b) $\eta^{\prime}=0.009<\eta_{c}^{\prime}$

a critical level of noise $\left(\eta>\eta_{c}\right)$, the curve reaches only one maximum whatever the value of $\varepsilon$ [see Fig. 12(a)]. In this case, the probability distribution has a wide bell shape, meaning that not a potential barrier no longer exists between the states. The most probable amplitude satisfies the relation

$$
\varepsilon^{\prime}=-\left|a_{\max }\right|^{2}+\left|a_{\max }\right|^{4}-\frac{\eta^{\prime}}{2\left|a_{\max }\right|^{2}} .
$$

Figure 13 depicts, for several noise levels, the corresponding stochastic subcritical bifurcation diagrams. The respective hysteresis region is delimited by $\varepsilon^{\prime}$ up and $\varepsilon^{\prime}$ down (see Fig. 13). When noise intensity increases, these two quantities come closer until a critical value $\eta_{c} \equiv 0.07$, where the bistable zone disappears (see Fig. 13).

From Eq. (29), it is possible to obtained expansions of $\varepsilon^{\prime}$ up and $\varepsilon^{\prime}$ down, in the range of validity $\eta=\left[0 ; \eta_{c}\right]$ :

$$
\begin{aligned}
\varepsilon_{\text {up }}^{\prime} & \approx-\frac{1}{4}-\eta^{\prime}-\eta^{\prime 2}-4 \eta^{\prime 3}, \\
\varepsilon_{\text {down }}^{\prime} & \approx-\sqrt{2} \eta^{\prime \frac{1}{2}}+\frac{1}{2} \eta^{\prime}+\frac{\sqrt{2}}{4} \eta^{\prime \frac{3}{2}}+\frac{1}{2} \eta^{\prime 2}-\frac{\sqrt{2}}{8} \eta^{\prime \frac{5}{2}}+\frac{1}{8} \eta^{\prime 3} .
\end{aligned}
$$

Their evolution with the level of noise is plotted in Fig. 14. This figure also shows the evolution of the Maxwell point $\varepsilon_{\mathcal{M}}\left(\eta^{\prime}\right)$ as function of noise intensity level. The Maxwell point is defined as the point where the two states have the same probability [see the inset of Fig. 14(c)]. In other words, at this particular value of $\epsilon$, the mean time of each equilibrium is equal. Imposing both maxima have the same probability, and we can estimate

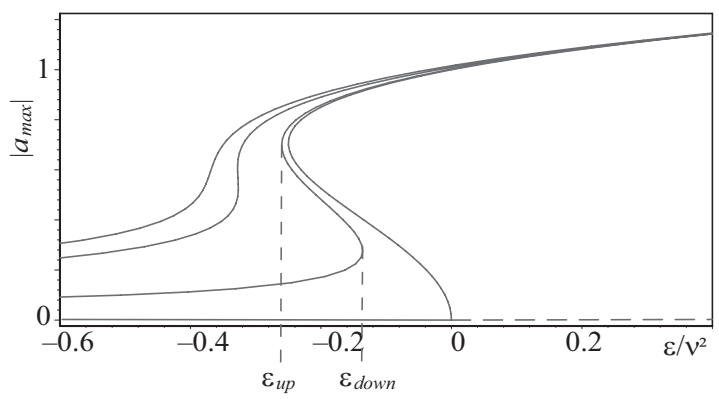

FIG. 13. Theoretical bifurcation diagram of the subcritical amplitude equation for different levels of noise. From right to left: $\eta^{\prime}=0 ; \eta^{\prime}=0.01<\eta_{c} ; \eta^{\prime}=\eta_{c} \equiv 0.07 ; \eta^{\prime}=0.1>\eta_{c} . \varepsilon^{\prime}$ down and $\varepsilon^{\prime}$ up account for the region of bistability for noise intensity level $\eta^{\prime}=0.01$.

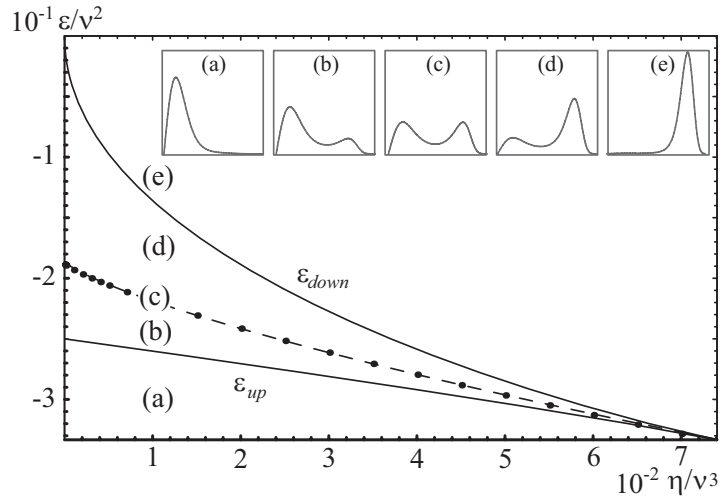

FIG. 14. Theoretical evolution of the bistability zone limits $\varepsilon_{\text {down }}$ (continued curve above) and $\varepsilon_{\text {up }}$ (continued curve below) with the level of noise. The intermediate curve represents the approximated evolution of the Maxwell point [Eq. (30)]. The dots are exact values of the Maxwell point numerically calculated. The shaded area represents the region of bistability. The insets represent, respectively, the generalized Rayleigh distribution in the respective places.

the maxwell point as

$$
\varepsilon_{\mathcal{M}} \approx-\frac{3}{16}+\frac{2}{3} \sigma \log (\sigma)\left[1+\frac{16 \sigma}{9}\right] .
$$

Notice that the decrease of $\varepsilon_{\text {up }}$, as a consequence of increasing noise intensity level, generates the decrease of the nucleation barrier between equilibria. For instance, taking $\varepsilon=$ -0.27 and increasing $\eta^{\prime}$ (which corresponds to a horizontal displacement in Fig. 14), the system is first in the state "down" to $\eta^{\prime} \approx 0.02$. This is the only state of equilibrium in this region. It then enters a bistable regime, crosses the Maxwell point, and, finally, reaches the state "up" when $\eta^{\prime} \gtrsim 0.044$. In the bistability region, we observe the phenomenon of noise induced transitions between the two equilibria.

\section{Numerical results}

In order to emphasize the former results we consider the subcritical Swift-Hohenberg equation [14],

$$
\partial_{t} u=\epsilon u+v u^{3}-u^{5}+\left(\partial_{x x}+q^{2}\right)^{2} u+\sqrt{\sigma_{0}} \xi(x, t) .
$$

Introducing a similar ansatz to (4) and after straightforward calculations, one comes across Eq. (8) again, with $b=3 v$, $h=10$, and $\eta^{\prime}=2 \sigma_{0} \pi / L$. Introducing appropriate scalings for the amplitude and time, this leads to Eq. (27).

Figure 15 shows the bifurcation diagrams of $\left|a_{\max }\right|$ versus $\varepsilon$ obtained from numerical simulations of Eq. (31) with different values of the noise intensity level $\sigma_{0}$. The solid curves are fits following the formula Eq. (29), except for relatively high noise intensities. In this latter case, two extra parameters $\{f, g\}$ have to be added to the previous fitting formula to remain valid,

$$
\varepsilon=-f\left|a_{\max }\right|^{2}+g\left|a_{\max }\right|^{4}-\frac{\eta^{\prime}}{2\left|a_{\max }\right|^{2}} .
$$

These extra coefficients compensate stochastic terms that were neglected to obtain the amplitude equation (27). Indeed, as the noise intensity increases, these terms become more relevant and no longer can be neglected. This is clearly stated, if one considers the stochastic amplitude Eq. (27). For large $\varepsilon$ values, 


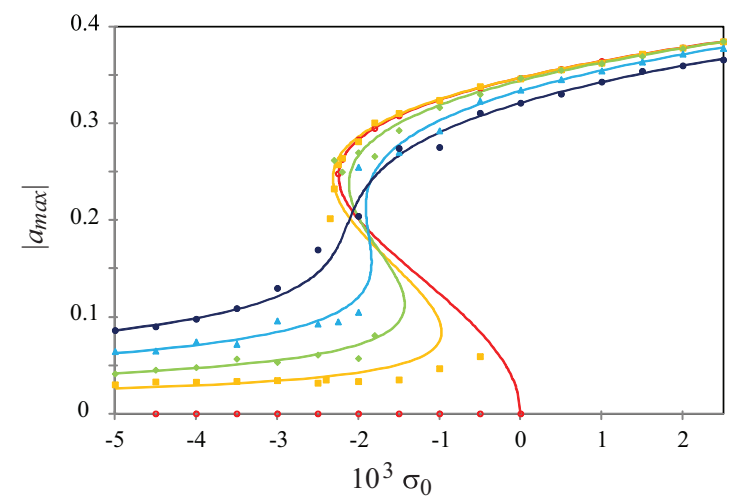

FIG. 15. (Color online) Numerical bifurcation diagram of the subcritical Swift-Hohenberg model, Eq. (31), for different levels of noise. Taking the curves of the left side from top to bottom $\sigma_{0}=0.1$ (blue •), $\sigma_{0}=0.05$ (cyan $\left.\boldsymbol{\Delta}\right), \sigma_{0}=0.02($ green $\bullet), \sigma_{0}=0.01$ (orange $\mathbf{\square}$ ), and $\sigma_{0}=0.0$ (red $\circ$ ). The respective solid curves are obtained using the fitting formula (32).

the bifurcation diagrams converge asymptotically to the same curve (see Fig. 13). We then can notice that, for the highest noise level, the bifurcations are still perfectly reproduced by the expression of Eq. (32) (Fig. 15). As can be seen from Fig. 13, we show how the noise can change the shape of the bifurcation diagram and even modify a transition from first- to second-order type for the Swift-Hohenberg model [Eq. (31)]. Hence, the noise can suppress the hysteresis cycle. This phenomenon has been explored in different models of subcritical bifurcations in extended systems (see the review in Ref. [19] and references therein).

\section{CONCLUSIONS}

In conclusion, we have given analytical expressions of stochastic super- and subcritical bifurcations obtained when additive noise is present in one-dimensional spatial systems.
A cubic and a quintic amplitude equation accounting for stochastic supercritical bifurcation and a cubic-quintic amplitude equation accounting for stochastic subcritical bifurcation have been considered. From the universal expressions of the most probable amplitude-which is most suitable variable that inherits the properties from the bifurcations of deterministic systems - the shapes of the bifurcations, their locations, and their evolutions with the noise level are completely defined. Thus, these expressions still allow the use of bifurcation diagrams to study and characterize classes of stochastic amplitude equations. Our predictions are compared with one experimental work and clearly provide evidence that the expression is relevant for describing the associated supercritical cases. Finally, we showed, in the subcritical case, that the level of additive fluctuations can change the type of the stochastic bifurcation from a sub- to a supercritical one.

The study of stochastic system dynamics is a complex work. However, a great advantage of studying their noisy bifurcations is that these latter can be modeled in an unified way by stochastic amplitude equations that usually have extra symmetries as compared to the original systems. Indeed, in the case of emergence of stationary patterns, the dynamics in the vicinity of bifurcation is characterized by a variational dynamics. This property allows us to derive a free energy or nonequilibrium potential and, in turn, to obtain simple expressions for bifurcation diagrams.

\section{ACKNOWLEDGMENTS}

The authors acknowledge financial support by ANRCONICYT 39 (ANR-2010-INTB-402-02), “Colors.” M.G.C. thanks FONDECYT Project No. 1120320 for support. R.G.R and E.L. acknowledge financial support by ECOS-Sud Committee, C10E04. This research was supported in part by the center de la Recherche Scientifique (CNRS) and by the "Conseil Régional Nord-Pas de Calais" and "The Fonds Européen de développment Economique de régions."
[1] G. Nicolis and I. Prigogine, Self-Organization in NonEquilibrium Systems (J. Wiley \& Sons, New York, 1977).

[2] L. Landau and E. Lifshitz, Statistical Physics (Pergamon press, New York, 1980), Chap. XIV.

[3] M. C. Cross and P. C. Hohenberg, Rev. Mod. Phys. 65, 851 (1993).

[4] V. Castets, E. Dulos, J. Boissonade, and P. De Kepper, Phys. Rev. Lett. 64, 2953 (1990).

[5] V. Barelko, Self-organization, Autowaves and Structures far from Equilibrium (Springer-Verlag, Berlin, 1984).

[6] Yu. Astrov, E. Ammelt, S. Teperick, and H.-G. Purwins, Phys. Lett. A 211, 184 (1996).

[7] J. R. Tredicce, E. J. Quel, A. M. Ghazzawi, C. Green, M. A. Pernigo, L. M. Narducci, and L. A. Lugiato, Phys. Rev. Lett. 62, 1274 (1989).

[8] F. Lonberg, and R. B. Meyer, Phys. Rev. Lett. 55, 718 (1985).

[9] C. Chevallard and M. G. Clerc, Phys. Rev. E 65, 011708 (2001).

[10] E. Koschmieder, Adv. Chem. Phys. 32, 109 (1975).
[11] E. A. Buka and L. Kramer, Pattern Formation in Liquid Crystal (Springer-Verlag, New York, 1996).

[12] L. Pismen, Patterns and Interfaces in Dissipative Dynamics (Springer-Verlag, Berlin, 2002).

[13] M. I. Rabinovich, A. B. Ezersky, and P. D. Weidman, The Dynamics of Patterns (World Scientific, Singapore, 2000).

[14] M. Cross and H. Greenside, Pattern Formation and Dynamics in Nonequilibrium Systems (Cambridge University Press, New York, 2009).

[15] P. Ball, The Self-Made Tapestry: Pattern Formation in Nature (Oxford University Press, New York, 1999).

[16] C. Meunier and A. Verga, J. Stat. Phys. 50, 345 (1988).

[17] L. Arnold, Random Dynamical Systems, Springer Monographs in Mathematics (Springer-Verlag, Berlin, 1998).

[18] N. Berglund and B. Gentz, Noise-Induced Phenomena in Slow-Fast Dynamical Systems (Springer-Verlag, London, 2006).

[19] J. Garcia-Ojalvo and J. M. Sancho, Noise in Spatially Extended Systems (Springer-Verlag, New York, 1999). 
[20] G. Agez, M. G. Clerc, and E. Louvergneaux, Phys. Rev. E. 77, 026218 (2008)

[21] R. Müller, K. Lippert, A. Kühnel, and U. Behn, Phys. Rev. E 56, 2658 (1997).

[22] R. Berthet, A. Petrossian, S. Residori, B. Roman, and S. Fauve, Physica D 174, 84 (2003).

[23] G. Agez, C. Szwaj, E. Louvergneaux, and P. Glorieux, Phys. Rev. A 66, 063805 (2002).

[24] C. Elphick, E. Tirapegui, M. Brachet, P. Coullet, and G. Iooss, Physica D 29, 95 (1987).

[25] M. Haragus and G.Iooss, Local Bifurcations, Center Manifolds, and Normal Forms in Infinite Dimensional Systems (EDP Sciences, Springer-Verlag, Heidelberg, 2011).

[26] P. Manneville, Dissipative Structures and Weak Turbulence (Academic Press, USA, Saclay, 1990).

[27] N. G. V. Kampen, Stochastic Process in Physics and Chemistry (North-Holland, Amsterdam, 1992).
[28] C. Gardiner, Handbook of Stochastic Methods (Springer-Verlag, Berlin, 1985).

[29] I. Ortega, M. G. Clerc, C. Falcón, and N. Mujica, Phys. Rev. E 81, 046208 (2010).

[30] I. Rehberg, S. Rasenat, M. de la Torre Juarez, W. Schopf, F. Horner, G. Ahlers, and H. R. Brand, Phys. Rev. Lett. 67, 596 (1991).

[31] D. Goldman, J. B. Swift., and H. L. Swinney, Phys. Rev. Lett. 92, 174302 (2004).

[32] G. D'Alessandro and W. J. Firth, Phys. Rev. A 46, 537 (1992).

[33] P. Coullet, T. Frisch, and G. Sonnino, Phys. Rev. E 49, 2087 (1994).

[34] M. G. Clerc, S. Coulibaly, and D. Laroze, Phys. Rev. E 77, 056209 (2008).

[35] F. Pétrélis, S. Aumaître, and S. Fauve, Phys. Rev. Lett. 94, 070603 (2005). 